\title{
A Mini-review of Culicoides (Diptera: Ceratopogonidae) Fauna and its Vectorial Role in Tunisia
}

\section{Ahmed Tabbabi}

Department of Hygiene and Environmental Protection, Ministry of Public Health, Tunis, Tunisia

\begin{abstract}
Following the bluetongue outbreaks in Tunisia from 1999 to 2006, bluetongue virus serotype 2 was isolated and several entomological investigations were performed. The present study reviewed the current status of Culicoides fauna in Tunisia and their role as proven and potential vectors of bluetongue virus based on the available and scattered reports. In total, about 35 species of Culicoides are known to occur in the country. Culicoides imicola is considered as the main and the proven vector of bluetongue virus. Other species of the genus Culicoides were suggested as potential vectors bluetongue virus. We cited mainly Culicoides obsoletus, Culicoides scoticus, Culicoides dewulfi and Culicoides pulicaris. Priority recommendations to prepare the country for future veterinary health challenges were suggested.
\end{abstract}

Keywords: Culicoides imicola; Bluetongue virus; Veterinary health; Tunisia

\section{Introduction}

Biting midges of the genus Culicoides (Diptera: Ceratopogonidae) are hematophagous insects found in tropical and temperate countries. These insects have an importance veterinary health importance by transmitting several pathogens $[1,2]$. Actually, about 1316 biting midges species have been identified and 50 arboviruses have been found inside species of Culicoides considered later as vectors [3,4]. Recently, Schmallenberg bunyavirus (genus Orthobunyavirus; Bunyaviridae) have been isolated in Culicoides species in Europe [5,6]. The aim of the present study was to review the species composition of Culicoides fauna in Tunisia and their role as proven and potential vectors of bluetongue virus.

\section{Current Species Composition of Culicoides Fauna in Tunisia}

Entomological investigations carried out in Tunisia have reported an increasing number of species during the last decades. In total, about 35 species of Culicoides are actually present in the country [711]: Culicoides imicola kieffer, 1913; Culicoides obsoletus Meigen, 1818; Culicoides circumscriptus Kieffer, 1918; Culicoides newsteadi Austen, 1921; Culicoides punctatus Meigen, 1804; Culicoides parroti Kieffer, 1922; Culicoides puncticollis Becker, 1903; Culicoides riethi Kieffer, 1914; Culicoides cataneii Clastrier, 1957; Culicoides corsicus Kremer, 1971; Culicoides gejgelensis Dzhafarov, 1964; Culicoides griseidorsum Kieffer, 1918; Culicoides heteroclitus Kremer and Callot,1965; Culicoides jumineri Callot and Kremer, 1969; Culicoides longipennis Khalaf, 1957b; Culicoides maritimus Kieffer, 1924; Culicoides pseudopallidus Khalaf, 1961; Culicoides santonicus Callot, Kremer, Rault and Bach, 1966; Culicoides semimaculatus Clastrier 1958a; Culicoides sergenti Kieffer, 1921h; Culicoides submaritimus=C. maritimus Borkent 2008; Culicoides univittatus Vimmer, 1932; Culicoides saevus Kieffer, 1922g; Culicoides kingi Austen, 1912; Culicoides fascipennis Staeger, 1839; Culicoides subfasciipennis Kieffer, 1919a; Culicoides sahariensis Kieffer, 1923a; Culicoides kurensis Dzhafarov, 1960; Culicoides langeroni Kieffer, 1921; Culicoides lailae A; Culicoides lailae $B=C$. odiatus Borkent 2008; Culicoides marcleti Callot, Kremer and Basset, 1968; Culicoides odiatus Austen 1921; Culicoides indistinctus=C. odiatus Borkent, 2008; Culicoides paolae Boorman, 1996.

\section{Vectorial Role of Culicoides as Proven and Potential Vectors in Tunisia}

Culicoides imicola is considered as the main and the proven vector of bluetongue virus and African horse sickness virus viruses Africa, Middle
East, southern Asia and southern Europe [12,13]. The two pathogens are responsible of devastating diseases in ovine and equidae, respectively. It is important to note that other species including Culicoides obsoletus, Culicoides scoticus, Culicoides dewulfi and Culicoides pulicaris are considered as known or potential bluetongue virus vectors [14-20]. In Tunisia, the first outbreaks of bluetongue virus (BTV) were identified in 1999 causing a dramatic sanitary and economical crisis in the country [21]. Later, two serotypes including BTV-2 and BTV-1 have arisen in 2000 and 2006, respectively [8]. In 1999, the first outbreak appeared in eastern Tunisia along the coast. An important rate of morbidity and mortality rates were recorded reaching $8.35 \%$ and $5.5 \%$ respectively [22]. One year later, 72 outbreaks affecting 6,120 sheep were recorded in eastern and central Tunisia. Vaccination campaigns were undertaken in sheep flocks by veterinary health authorities in order to control the propagation of the disease.

\section{Priority Recommendations to Prepare the Country for Future Veterinary Health Challenges}

Control against bluetongue disease consists to ameliorate our knowledge of the epidemiology of blue tongue and to better understand the vectors ecology. The dominance of Culicoides imicola compared to other collected species highlights the risk of emergence of new diseases. Further entomological investigations are essential to continue the surveillance of other potential and to limit the propagation of the disease in the country. Understanding what a vector is to evaluate and predict vector transmission risks, improve current control methods and develop new approaches and Coordinating multidisciplinary research efforts are the main and priority recommendations to prepare the country for future veterinary health challenges.

\section{References}

1. Borkent A (2005) The biting midges, the Ceratopogonidae (Diptera). In Marquardt W.C. (ed). Biology of Disease Vectors pp: 113-126.

*Corresponding author: Ahmed Tabbabi, Department of Hygiene and Environmental Protection, Ministry of Public Health, Tunis, Tunisia, Tel: +0021697085424; E-mail: tabbabiahmed@gmail.com

Received November 24 2017; Accepted December 06, 2017; Published December 11, 2017

Citation: Tabbabi A (2017) A Mini-review of Culicoides (Diptera: Ceratopogonidae) Fauna and its Vectorial Role in Tunisia. Hereditary Genet 6: 189. doi:10.4172/2161 1041.1000189

Copyright: ( 2017 Tabbabi A. This is an open-access article distributed under the terms of the Creative Commons Attribution License, which permits unrestricted use, distribution, and reproduction in any medium, provided the original author and source are credited. 
Citation: Tabbabi A (2017) A Mini-review of Culicoides (Diptera: Ceratopogonidae) Fauna and its Vectorial Role in Tunisia. Hereditary Genet 6: 189. doi:10.4172/2161-1041.1000189

Page 2 of 2

2. Meiswinkel R, Venter GJ, Nevill EM (2004) Vectors: Culicoides spp. Infectious diseases of livestock pp: 93-136.

3. Borkent A (2011) Numbers of extant and fossil species of Ceratopogonidae. Illinois Natural History Survey, University of Illinois, USA.

4. Meiswinkel R, Labuschagne K, Goffredo M (2004) Culicoides: C. Jamaicans Introduction into the Mediterranean and 500 years, later renamed as C. paolae. 3rd International Symposium, Taormina.

5. Rasmussen LD, Kristensen B, Kirkeby C, Rasmussen TB, Belsham GJ, et al. (2012) Culicoids as vectors of Schmallenberg virus. Emerg Infect Dis 18: 1204 1206.

6. Eagles D, Walker PJ, Zalucki MP, Durr PA (2013) Modelling spatiotemporal patterns of long-distance Culicoides dispersal into northern Australia. Prev Vet Med 110: 312-322.

7. Chaker E, Kremer M (1982) Culicoides of Tunisia: Morphological characteristics. Chorology and ecology of species found. Arch Inst Past T 59: 511-540.

8. Sghaier S, Hammami S, Hammami H, Dkhil A, Delécolle JC (2009) Entomological surveillance of Culicoides (Diptera: Ceratopogonidae), vector of Bluetongue in Tunisia. Rev Elev Méd Vété Pays Trop p: 62.

9. Chaker E, Sfari M, Rabhi M, Rouis M, Babba H, et al. (2005) Faunistic note on Culicoides (Diptera, Ceratopogonidae) from the governate of Monastir (Tunisia). Parasite 12: 359-361

10. Hammami S, Bouzid M, Hammou F, Fakhfakh E, Delecolle JC (2008) Occurrence of Culicoides spp. (Diptera: Ceratopogonidae) in Tunisia, with emphasis on the bluetongue vector Culicoides imicola. Parasite 15: 179-181.

11. Slama D, Chaker E, Zrelli S, Mathieu B, Delecolle JC, et al. (2016) Culicoides (Diptera: Ceratopogonidae) Fauna in Central Tunisia. Entomol Ornithol Herpetol 5: 184

12. Mellor PS, Boorman J (1995) The transmission and geographical spread of African horse sickness and bluetongue viruses. Annals of Tropical Medicine and Parasitology 89: 1-15.
13. Mellor PS, Wittmann EJ (2002) Bluetongue virus in the Mediterranean basin 1998-2001. The Veterinary Journal 164: 20-37.

14. De Liberato C, Scavia G, Lorenzetti R, Scaramozzino P, Amaddeo D, et al (2005) Identification of Culicoides obsoletus (Diptera: Ceratopogonidae) as a vector of bluetongue virus in Central Italy. Vet Rec 156: 301-304.

15. Mehlhorn H,Walldorf V, Klimpel S, Jahn B, Jaeger F, et al. (2007) First occurrence of Culicoides obsoletus-transmitted bluetongue virus epidemic in central Europe. Parasitol Res 101: 219-228.

16. Mehlhorn H, Walldorf V, Klimpel S, Schaub G, Kiel E, et al. (2009) Bluetongue disease in Germany (2007-2008): monitoring of entomological aspects. Parasitol Res 105: 313-319.

17. Meiswinkel R, Van Rijn P, Leijs P, Goffredo M (2007) Potential new Culicoides vector of bluetongue virus in northern Europe. Vet Rec 161: 564-565.

18. Meiswinkel R, Baldet T, De Deken R, Takken W, Delécolle JC, et al. (2008) The 2006 outbreak of bluetongue in northern Europe-the entomological perspective. Preventive Vet Med 87: 55-63.

19. Calvete C, Estrada R, Miranda MA, Borras D, Calvo JH, et al. (2008) Modelling the distributions and spatial coincidence of bluetongue vectors Culicoides imicola and the Culicoides obsoletus group throughout the Iberian Peninsula. Med Vet Entomol 22:124-134.

20. Nielsen SA, Nielsen BO, Chirico J (2010) Monitoring of biting midges (Diptera: Ceratopogonidae: Culicoides latreille) on farms in Sweden during the emergence of the 2008 epidemic of bluetongue. Parasitol Res 106: 1197-1203.

21. Ben Fredj S, Bréard E, Sailleau C, Zientara S, Zekri S, et al. (2003) Incursion de la fièvre catarrhale ovine en Tunisie: caractérisation moléculaire des isolats viraux. Elev Méd Vét Pays Trop 56: 121-127.

22. Hammai S (2003) North Africa: A regional overview of bluetongue virus vectors, surveillance and unique features. Bluetongue, proceedings of the third international symposium. $26^{\text {th }}$ to $29^{\text {th }}$ October. Taormina, Italy, $40: 43-46$. 